possible unequal illumination, for the nearer edge of the plate necessarily receives more light than the more distant one. The "plastic" illustrating this article (Fig. 1) was exposed fifteen minutes on bromid paper.

When developed the print (No. 3) will show beautiful "plastic" effects and the soft parts usually stand out in wonderful clearness. The more perfect the first negative-Plate No. 1-the better the "plastic."

How does this "plastic" result from the double exposure and the combination of the negatives? We do not know. According to the law and the prophets the combination of an equally dense and perfectly centered negative and positive should result in an absolute blank, as the opacities in the one and the transparent parts of the other should counterbalance.

How does the "plastic" differ from the ordinary skiagram? As previously stated, the "plastic" does not consist merely of the overlapping of half-tones of different intensity, but contains "shadows and high lights" -the two light values which make an ordinary photograph or drawing stand out in relief.

Are the effects mere photograph dodges? So the skeptics have claimed, and at first sight we were inclined to the belief that they were mere artifacts. But one by one the skeptics have been silenced and have joined the ranks of the believers until even Schellenberg, the earliest and firmest opponent, has been converted and forced to admit the "plastic" radiography to the greatest advance since Roentgen.

As for the theories and rationale of "shadowgraphs and plastics" and the explanation of the various phenomena involved in their production, we are not yet able to speak from conviction. It has taken most of our time to perfect the details, and we shall give at another time and in a more suitable place an explanation of the various principles involved.

\section{A SIMPLE AND SAFE OPERATION ON THE FRONTAL SINUS BY THE INTRA-NASAL ROUTE.}

\section{R. H. GOOD, M.S., M.D. CHICAgO.}

The instruments used in this operation are two frontal sinus rasps, two tapering curved chisels and three protectors.

The frontal sinus rasp (Fig. 1) is made on the same

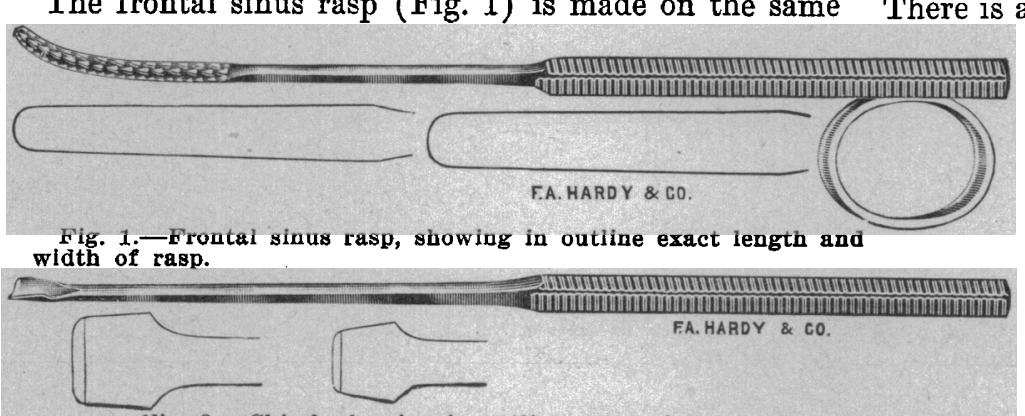

Fig. 2.-Chisel, showing in outline exact size.

principle as the old-fashioned coarse horseshoe rasp, from which I derived the idea. It is slightly curved at the rasp end to fit into the frontal sinus and the teeth are on the longitudinally concave surface, pointing toward the handle, so that when traction is made on the rasp, the bone anterior to it is rasped away, as illustrated in Figure 5. The posterior surface of the rasp is perfectly smooth and flat.
The chisels (Fig. 2) are short, curved and tapering. The handle at the chisel end is very slender so that the chisel can be manipulated under the direct vision of the eye. The edges are tapering as well as blunt, so that if the edge should strike a bony surface it would tend to glide off instead of penetrating.

The protectors (Fig. 3) are made of brass and in three different widths, as illustrated. They can be bent easily into shape on the flat, but not sideways.

$I$ have performed these operations under local anesthesia. The cocain crystals are rubbed into the mucous'membrane with a cotton swab on an applicator

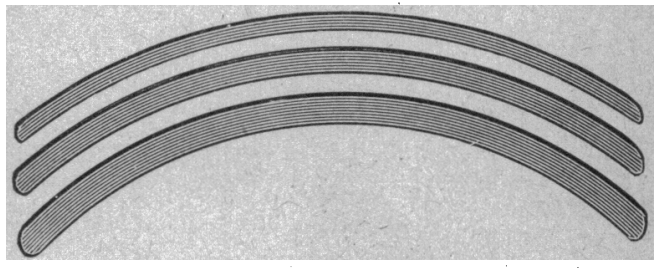

Fig. 3.-Frontal sinus protectors.

which is first moistened with adrenalin chlorid 1 to 1,000 , and then dipped into the cocain crystals. The entire olfactory region as well as the bulla ethmoidalis and unciform plate are thus anesthetized. The middle turbinate is then removed by the ordinary methods and the processus uncinatus of the ethmoid bone is removed with the larger chisel:

A frontal sinus catheter, with syringe attachment, is then inserted into the frontal sinus and a half dram of a 10 per cent. solution of cocain in adrenalin 1 to 1,000 is injected into the sinus. A pledget of cotton is placed over the infundibulum for a few minutes to take up the cocain as it returns from the sinus. The largest possible protector is now inserted into the frontal sinus, and if the smallest protector will not enter, which is rare, a good-sized probe is used, until later in the operation when the protectors can be inserted.

Having the protector or probe, as the case may be, in situ, the larger chisel is placed in position as illustrated in Figure 4, and a part of the processus frontalis of the superior maxillary bone, as well as a part of the spina frontalis of the frontal bone, and the anterior medial wall of the labyrinthus ethmoidalis are chiseled through and removed with forceps or curettes.

The chisel must always be kept in direct line with the protector so that the tabula interna is not endangered. There is a considerable space between the spina frontalis and the tabula interna of the frontal bone, and the operator can almost always chisel without touching the protector, for the moment the chisel has chipped off a piece of bone it has to pass through a space before it encounters the protector, and the hand nearly always will stop the chisel before it touches the protector; and in case it does strike the protector, the hand has reduced the force to such an extent as to make hardly a mark on the protector.

As stated above, the edges of the chisel are dull and tapering, so that if the edge should touch the lamina papyracea of the ethmoid bone or the tabula interna of the frontal bone, it will slide off and by the time the chisel has traveled through the space of about half an inch, under proper care, would not have enough force to penetrate even if the edges were sharp.

The anterior ethmoidal cells are removed with forceps or curette, and by this time the frontal sinus rasp may 
be introduced into the sinus; and if not, the smaller chisel is used to make the opening a little larger. When using the small chisel high up I put in two protectors at a little different angle to insure still better protection.

The structures to be guarded against injury are the lachrymal bone, lamina papyracea and the inner lower floor of the frontal sinus. By keeping a prepared skull in front of you while operating it is almost impossible to penetrate these structures, but an assistant can put his finger into the orbit and can tell at once whether the chisel is lifting the orbital periosteum. The lamina cribrosa is not in danger because the chisel is directed outward and not in the direction of this structure. The tabula interna is protected first by the protectors, and

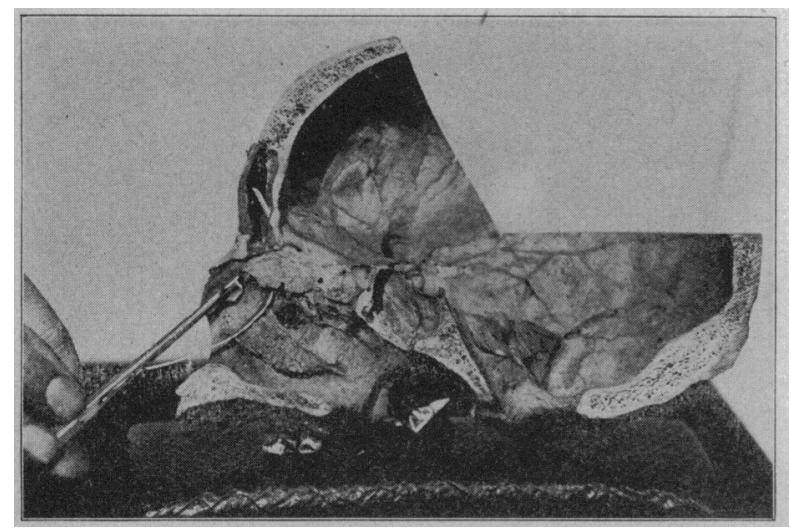

Fig. 4.- Showing chisel and protector in position.

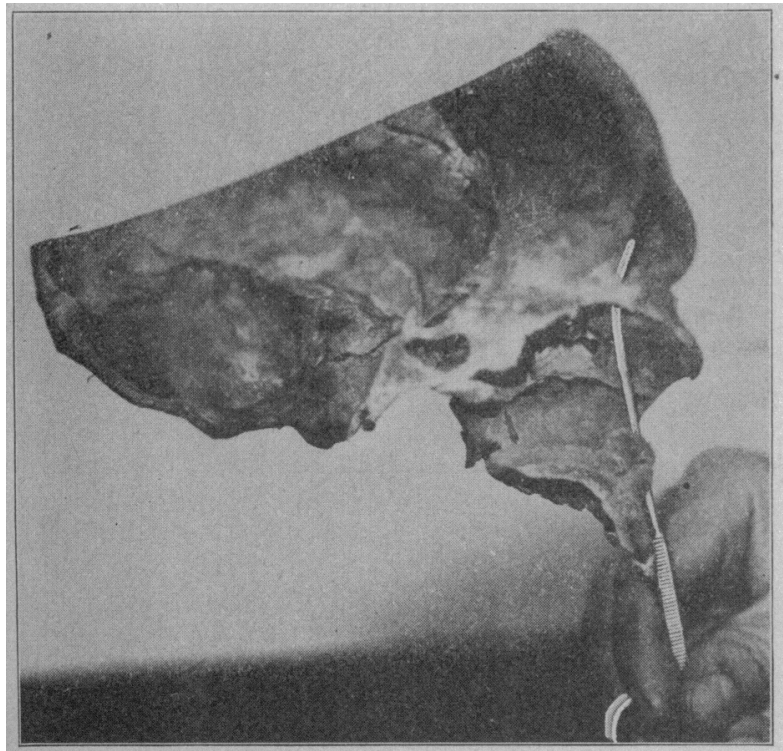

Fig. 5.-Frontal sinus rasp in position.

second by the fact that the chisel has to go through a space before touching it, and being carefully held by the hand, loses its momentum by the time it reaches this structure.

The frontal sinus rasp is now easily introduced, as illustrated in Figure 5. With this instrument the spina frontalis is rasped out, as well as the frontal accessory ethmoidal cells, if present (Fig. 6). There is absolutely no danger to the intracranial structures, and the worst thing that could happen would be to rasp through the orbital wall, but this can be avoided by an assistant putting his finger into the orbit.
The rasp must not be directed directly forward, but outward and forward, and care must be taken not to withdraw the rasp too far, as it will damage the septum and cause synechia between it and outer wall, as occurred in one of my cases, which was, however, easily remedied. The sinus is then curetted with flexible cur-ettes and diseased membrane removed. The reaction following this operation is very slight, and patients who have been suffering pain for several weeks will sleep with comfort the first night after the operation.

The sinus is packed with sterile gauze, soaked in carbolized petrolatum, to which has been added a few drops of oil of cloves. In twenty-four hours the packing is removed, and after that the patient is left absolutely alone, except that care should be taken that no adhesions form between the septum and the outer wall; if granulations form, cauterize them with silver nitrate.

I believe that my frontal sinus rasp would make a valuable addition to Dr. Ingal's method of making an opening into the sinus with a burr, as the rasp would fit into the opening which his burr drills out, and thus the opening could be enlarged three or four times. I feel that my chisels are less dangerous than the drill.

I have had the pleasure of seeing some of Dr. Halle's patients operated on by his drill method, and he makes

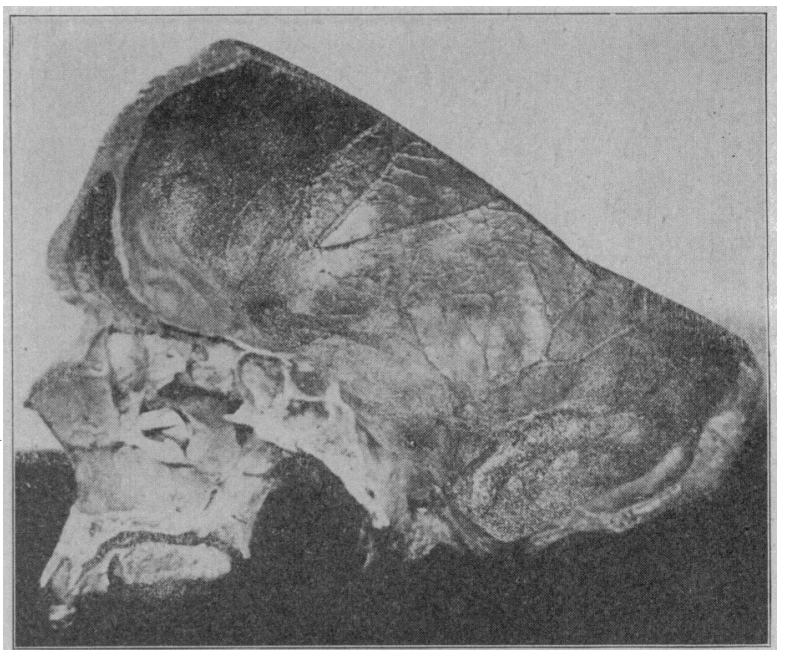

Fig. 6.-Showing size of opening when operation is completed. The ethmoid cells are all removed in this preparation.

a good large opening. I, however, consider my method much simpler, requiring but a cheap instrumentarium, and the operation can be done in a much shorter time. My experience with drills, run by motor power, is that it is more or less difficult to keep them absolutely under control, whereas my rasp can be used without any danger whatever to the intracranial structures.

I believe that with this method a large majority of the frontal sinusitis cases can be cured without resort to the external disfiguring operation. This operation should be done in all cases where an external operation is indicated, as it does no harm and is of great assistance in diagnosing cholesteatoma or tumors in the sinus, and should an external operation be necessary, a good drainage has already been established of larger size than is ordinarily made when doing the external operation.

Cool Temperature Checks Mosquito Biting.-Dr. Carlos J Finlay, ehief sanitary officer of Havana province, thinks that in Havana a daily mean temperature below $23 \mathrm{C}$. $(73.4 \mathrm{~F}$.) does not allow the stegomyia to bite and suck blood. 